\title{
Identification of a CAP (adenylyl-cyclase- associated protein) homologous gene in Lentinus edodes and its functional complementation of yeast CAP mutants
}

\author{
Guo-Lei Zhou, ${ }^{1}$ Yasumasa Miyazaki, ${ }^{2}$ Tsuyoshi Nakagawa, ${ }^{3}$ \\ Katsunori Tanaka, ${ }^{1}$ Kazuo Shishido, ${ }^{2}$ Hideyuki Matsuda ${ }^{1}$ \\ and Makoto Kawamukai ${ }^{1}$
}

Author for correspondence: Makoto Kawamukai. Tel: +8185232 6587. Fax: +81 852326499.
e-mail: kawamuka@life.shimane-u.ac.jp

1 Department of Life Science and Biotechnology, Faculty of Life and Environmental Science, Shimane University, Matsue 690, Japan

2 Department of Life Science, Faculty of Bioscience and Biotechnology, Tokyo Institute of Technology, Yokohama 226, Japan

3 Research Institute of Molecular Genetics, Shimane University, Matsue 690, Japan

\begin{abstract}
The adenylyl-cyclase-associated protein, CAP, was originally identified in yeasts as a protein that functions in both signal transduction and cytoskeletal organization. This paper reports the identification of a CDNA and genomic DNA that encodes a CAP homologue from the mushroom Lentinus edodes. The $L$. edodes cap gene contains eight introns and an ORF encoding a 518 amino acid protein. The $L$. edodes CAP is $35.5 \%$ and $\mathbf{4 0 . 9} \%$ identical at the amino acid level with Saccharomyces cerevisiae CAP and Schizosaccharomyces pombe CAP, respectively. The C-terminal domain shows greater homology (39-46\% identity) with yeast CAPs than does the $\mathrm{N}$-terminal domain (27-35\% identity). Southern blotting and Northern blotting results suggest that $L$. edodes cap is a single-copy gene and uniformly expressed. Expression of the $L$. edodes CAP in both Schiz. pombe and Sacch. cerevisiae complemented defects associated with the loss of the C-terminal domain function of the endogenous CAP. By using a yeast two-hybrid assay, an interaction was demonstrated between the $L$. edodes CAP and Schiz. pombe actin. This result and the functional complementation test indicate that CAP from $L$. edodes has a conserved Cterminal domain function.
\end{abstract}

Keywords: actin, adenylyl cyclase, cAMP, Ras

\section{INTRODUCTION}

The adenylyl-cyclase-associated protein (CAP) was simultaneously identified in the budding yeast Saccharomyces cerevisiae as an adenylyl-cyclase-binding protein with a molecular mass of $70 \mathrm{kDa}$, and as a mutated protein that suppresses the heat-shock-sensitive phenotype resulting from the activated $R A S 2^{\text {val19 }}$ mutation (Fedor-Chaiken et al., 1990; Field et al., 1990). Although genetic experiments implicate a function for CAP in RAS regulation of adenylyl cyclase in vivo, CAP is not necessarily required for the activation of adenylyl cyclase by RAS in vitro (Mintzer \& Field, 1994; Wang et al.,

\footnotetext{
Abbreviations: SH3, Src homology 3; CAP, adenylyl-cyclase-associated protein.

The GenBank/EMBL/DDBJ accession numbers for the sequence reported in this paper are $A B 001578$ and $A B 007849$.
}

1992). However, CAP may be involved in facilitating this interaction in vivo (Yu et al., 1994).

Deletion analysis has revealed that Sacch. cerevisiae CAP has two distinct functional domains. The Nterminal domain (amino acids 1-168) is required and sufficient for the $R A S 2^{\text {val19 }}$ responsiveness of adenylyl cyclase, while the widely conserved C-terminal region (amino acids 368-526) appears to play a role in the nutritional response and serves an important cytoskeletal regulatory function through the binding of monomeric actin (Gerst et al., 1991; Vojtek et al., 1991; Shima et al., 1997). Deletion of the C-terminal region results in abnormal phenotypes, including nutritional and temperature sensitivity, grossly enlarged cells, abnormal shape, random budding and a disorganized cytoskeleton (Field et al., 1990; Gerst et al., 1991). Expression of the C-terminal domain of CAP is sufficient to suppress these defects (Field et al., 1990; Vojtek et al., 
1991). These deficiencies could also be partially complemented by the overexpression of profilin in Sacch. cerevisiae (Vojtek et al., 1991). CAP and profilin can compensate some of each other's functions in Dictyostelium discoideum (Gottwald et al., 1996). The deletion of the centrally located, proline-rich domain (amino acids 169-367) of Sacch. cerevisiae CAP is phenotypically silent, but recently, the central region was identified as the $\mathrm{SH} 3$ (Src homology 3) binding region and was found to modulate cytoskeletal localization (Freeman et al., 1996). This region was also thought to be related to the localization of CAP in yeast (Zelicof $e t$ al., 1996).

Following the identification of CAP in Sacch. cerevisiae, CAP homologues were also identified in other eukaryotes, ranging from the fission yeast Schizosaccharomyces pombe to humans; two CAP homologues were found in humans and rat respectively (Kawamukai et al., 1992; Fenger et al., 1994; Vojtek \& Cooper, 1993; Zelicof et al., 1993; Gieselmann \& Mann, 1992; Matviw et al., 1992; Marcus et al., 1993; Yu et al., 1994; Gottwald et al., 1996; Swiston et al., 1995). Schiz. pombe CAP, which was the second CAP to be identified, has a similar function to Sacch. cerevisiae CAP. The Nterminal domain is associated with adenylyl cyclase binding, while deletion of the $\mathrm{C}$-terminal domain results in morphological and nutritional defects (Kawamukai $e t$ al., 1992). All of the homologous proteins identified share structural similarity and functional complementation. Structural similarity is greater in the C-terminal domains and central domains of CAPs than in the Nterminal domains. This is consistent with the observation that the expression of all tested CAP homologues in cap yeast strains can complement the defects resulting from the deletion of the C-terminal domain of endogenous CAP, but not those resulting from the deletion of the N-terminal domain (Kawamukai et al., 1992; Fenger et al., 1994; Vojtek \& Cooper, 1993; Zelicof $e t$ al., 1993; Gieselmann \& Mann, 1992; Matviw et al., 1992; Gottwald et al., 1996; Swiston et al., 1995). To investigate whether CAP and its functions have been conserved in mushrooms, we screened for a CAP homologue in the mushroom Lentinus edodes, strain FMC2. This mushroom is known to require high intracellular levels of cAMP activity for the onset of fruiting (Takagi et al., 1988) and to contain a homologue of the ras gene (Hori et al., 1991). Here we report the identification of the cap gene of L. edodes and show that it encodes a protein structurally and functionally homologous to yeast CAPs.

\section{METHODS}

Strains and media. Schiz. pombe strain MK1818d [h90 leu1-32 ade6-210 ura4-d18 cap::ura4 (cad11)] and Sacch. cerevisiae strain SKN32 [MATa leu2 ura3 trp1 ade8 can1 cap::HIS3] were used for the functional complementation test. Sacch. cerevisiae strain L40 [MATa bis 3 trp1 lew 2 ade 2 LYS2::lexA-HIS3 URA3::lexA-lacZ] was used for the twohybrid assay. Schiz. pombe was grown in either YEA rich medium $\left(0 \cdot 5 \%\right.$ yeast extract, $3 \%$ glucose, $75 \mathrm{mg}$ adenine $\left.\mathrm{l}^{-1}\right)$ or PMA (pombe minimal medium with $75 \mathrm{mg}$ adenine $\mathrm{l}^{-1}$ ) synthetic medium supplemented, when necessary, with $75 \mathrm{mg}$ leucine $l^{-1}$ (Mitchison, 1970). Sacch. cerevisiae was grown in YPD rich medium ( $1 \%$ yeast extract, $2 \%$ peptone, $2 \%$ glucose), or SC (synthetic complete) medium with appropriate auxotrophic supplements (Rose et al., 1990). The lithium acetate method was used for yeast transformation (Ito et al., 1983). The L. edodes strain FMC2 (Takagi et al., 1988) was used. Mycelia were grown in SMY medium (1\% sucrose, $1 \%$ malt extract, $0.4 \%$ yeast extract, $\mathrm{pH} 5 \cdot 6$ ). Fruiting bodies were formed according to the method previously described (Takagi et al., 1988).

DNA manipulation and analysis. General procedures for DNA manipulation and analysis were as described by Sambrook et al. (1989). L. edodes FMC2 genomic DNA was extracted from mycelia at the vegetative growth stage, as described by Zolan \& Pukkila (1986). The PCR kit was purchased from TaKaRa Biomedicals and was used as recommended by the supplier. Reaction mixtures in a total volume of $100 \mu \mathrm{l}$ were incubated for 30 cycles (a typical cycle was for $30 \mathrm{~s}$ at $94^{\circ} \mathrm{C}, 60 \mathrm{~s}$ at $52^{\circ} \mathrm{C}$ and $120 \mathrm{~s}$ at $\left.72^{\circ} \mathrm{C}\right)$. The annealing temperature and duration of each step varied slightly depending on the primers and the length of fragments to be amplified. The sequences of both strands of the DNA from the clones were determined by a modified dideoxy chain-termination method (Sanger et al., 1977) using the ABI sequencer Prism 377 and the Perkin Elmer Cycle-Sequencing kit. For amplification of a part of the cap gene, degenerate oligonucleotides complementary to peptides PPPPP and THKNP, which are two well-conserved motifs in most CAP homologues (amino acids 278-282 and amino acids 333-337 in Sacch. cerevisiae CAP), were designed and used as PCR primers. Using three forward primers and three reverse primers, nine possible primer combinations were tried in order to amplify the cap fragment by PCR. The primers used were as follows ( $\mathrm{N}$ represents any one of the four nucleotides). (1) Primers encoding THKNP (reverse primers): THK1, 5'GGGCGGCCGC(AC)GG(AG)T'T(TC)TT(GA)TG(AG)GT3'; THK2, 5'-AAGCGGCCGCACGTAAANNNGG(AG)TT(TC)TT(GA)TGNGT-3'; THK3， 5'-AAGCTT(AG)TT(TC)TT(GA)TG(ATG)GT-3'. (2) Primers encoding PPPPP (forward primers): P1, 5'-AAGCGGCCGC(AT)CC(AT)$\mathrm{CC}(\mathrm{AT}) \mathrm{CC}(\mathrm{AT}) \mathrm{CC}(\mathrm{AT}) \mathrm{CCT}-3^{\prime} ; \mathrm{P} 2,5^{\prime}$-GGATCCNCCNCC(TA) CC(TA)CC-3'; P3, 5'-AAGGATCCNCCNCC(TAC)$\mathrm{CC}(\mathrm{CG}) \mathrm{CC}-3^{\prime}$.

To amplify full-length cap from genomic DNA, primers were designed from the cap cDNA sequence (Fig. 2) and used in PCR. The forward and reverse primers were: Pfg (forward primer), TAAGCTTCGGCAGGATGTCCT; Prg (reverse primer), TGGATCCGATAATATGCATGT.

An $L$. edodes cDNA library was constructed by inserting the cDNA synthesized on poly (A) ${ }^{+}$RNA into an EcoRI-cut and dephosphorylated $\lambda$ gt10 vector (Huynh et al., 1985) according to the procedures previously described (Hori et al., 1991). $\operatorname{Poly}(\mathrm{A})^{+}$RNA was prepared from primordia and immature fruiting bodies.

Plasmids. Plasmid pMf9-6 was constructed by inserting a 196 bp NotI-NotI PCR fragment containing the cap sequence from the genomic DNA of L. edodes FMC2 into the Not I site of pBluescript II SK + . pMfcCAP was constructed by inserting a $1.7 \mathrm{~kb}$ EcoRI-EcoRI fragment containing cap cDNA into pBluescript II SK+ (Fig. 1). The cDNA was derived by screening a $\lambda \mathrm{gt} 10 \mathrm{~L}$. edodes cDNA library using plaque hybridization and the PCR fragment as a probe. pMfgCAP was constructed by inserting a $2 \cdot 1 \mathrm{~kb}$ PCR fragment containing cap genomic DNA into the pT7-Blue T-vector 

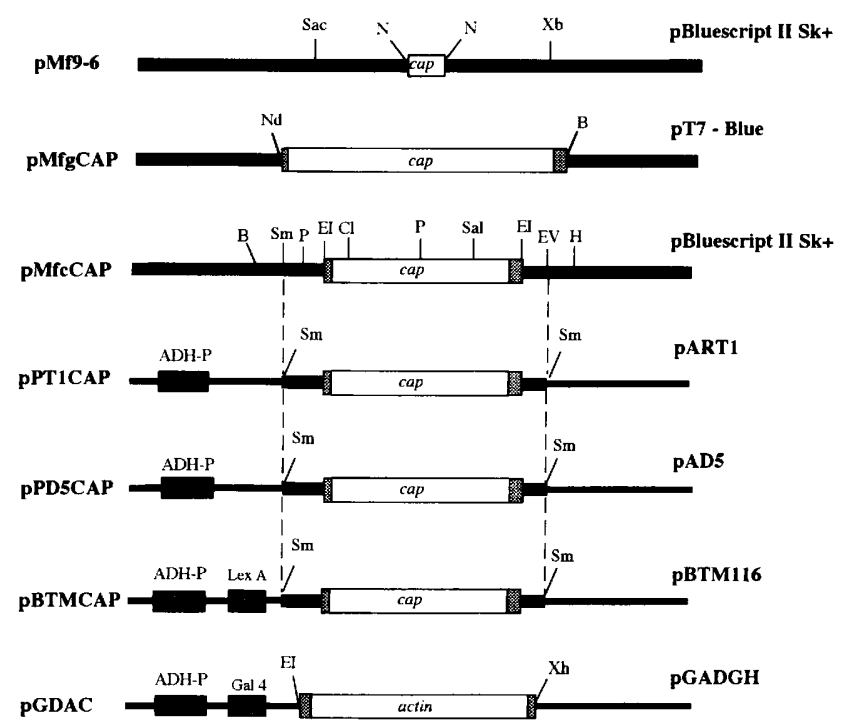

$1 \mathbf{k b}$

Fig. 1. Restriction maps of the plasmids used in this study. The construction of all plasmids is described in Methods. Open boxes indicate coding sequences of cap or other genes; stippled boxes represent the non-coding sequence flanking cap or actin genes derived from the CDNAs. The hatched boxes indicate the yeast $A D H 1$ promoter. The solid lines indicate the vector sequences: the thinner lines represent those derived from expression vectors or two-hybrid vectors, and the thicker lines represent those derived from the vector. Abbreviations for restriction enzymes are: $\mathrm{B}, \mathrm{BamHI} ; \mathrm{Cl}, \mathrm{Clal}$; El, EcoRI; EV, EcoRV; H, Hindlll; Nd, Ndel; N, Notl; P, Pstl; Sac, Sacll; Sal, Sall; Sm, Smal; Xb, Xbal; Xh, Xhol.

(Novagen). pPT1CAP was constructed by inserting the EcoRV-Smal fragment derived from pMfcCAP, which contains the cap cDNA, into the SmaI site of the Schiz. pombe expression vector pART1 (Mcleod et al., 1987). The fragment was placed in the correct direction for expression. The same fragment was inserted into the SmaI site of the Sacch. cerevisiae expression vector pAD5 (Kawamukai et al., 1992) to construct the plasmid pPD5CAP. Plasmid pCGE2 expresses Schiz. pombe CAP (Kawamukai et al., 1992). pADHCAP contains the Sacch. cerevisiae CAP coding sequence cloned into the SmaI site of pAD4, a plasmid similar to pADANS (Ballester et al., 1989; Field et al., 1990). For the two-hybrid assay, pBTMCAP was constructed by inserting the EcoRV-SmaI fragment containing cap cDNA into the SmaI site of pBTM116 (Bartel et al., 1993). The fragment was inserted in the correct frame so as to allow expression of a LexA-CAP fusion protein. Plasmid pGDAC was obtained from another two-hybrid screening; it contained the Schiz. pombe actin gene cloned into the EcoRI-Xhol site of pGADGH. The inserted fragment was confirmed to be in the correct frame for expression of the Gal4-fused protein. The plasmids are illustrated in Fig. 1.

Southern hybridization. Using the insert containing cap cDNA from plaque hybridization as a probe, we conducted Southern hybridization with $L$. edodes genomic DNA. The genomic DNA was first digested with several restriction enzymes, and then hybridization was performed under low-stringency conditions by using a washing buffer containing $0.5 \times$ SSC.
Northern-blot analysis. Total cellular RNA was isolated from primordia, immature fruiting bodies (stage I: just after formation of the pileus and stipe), immature fruiting bodies (stage II : fruiting bodies enlarged but gill tissues not yet visible), and mature fruiting bodies of $L$. edodes according to the method of Han et al. (1987). The RNA samples $(20 \mu \mathrm{g}$ each) were size-fractionated, transferred to a nylon filter, hybridized with ${ }^{32} \mathrm{P}$-labelled probes under stringent conditions according to the method previously described (Hori et al., 1991), and autoradiographed.

Yeast two-hybrid assay. Sacch. cerevisiae strain L 40 was cotransformed with pBTMCAP or its derivatives along with pGDAC or its derivatives, spread onto SC-Leu-Trp synthetic medium and grown for $2 \mathrm{~d}$ at $30^{\circ} \mathrm{C}$. The transformants were then plated onto a new SC-Leu-Trp plate covered with a piece of sterilized filter, further incubated for $1-2 \mathrm{~d}$ and tested for $\beta$ galactosidase activity by a filter assay (Fields \& Song, 1989).

\section{RESULTS}

\section{Cloning of $L$. edodes CDNAs encoding a CAP- homologous protein}

Part of the cap-homologous gene from $L$. edodes genomic DNA was amplified by PCR. Fragments of about $200 \mathrm{bp}$ or larger (considering the possibility of the existence of introns) were chosen, cloned into pBluescript II SK + , and their nucleotide sequences were determined. Among approximately 40 sequenced clones, one clone designated pMf9-6, derived from the primer combination THK2 and P1, was found to encode an amino acid sequence highly homologous to the corresponding region of other CAP proteins. The $196 \mathrm{bp}$ NotI-NotI fragment was labelled with ${ }^{32} \mathrm{P}$ and used as a probe to screen, by plaque hybridization, a $L$. edodes cDNA library previously constructed in the phage vector $\lambda$ gt10 using the EcoRI site (Hori et al., 1991). One positive clone designated G3 was obtained after screening about $2 \times 10^{5}$ plaques. The G3 insert was cut out by EcoRI digestion and subcloned into pBluescript II $\mathrm{SK}+$ to construct pMfcCAP (Fig. 1). The nucleotide sequence of G3 was determined. It contained an ORF encoding a protein of 518 aa that was structurally homologous to yeast CAP proteins and other homologues; therefore we designated the isolated gene cap. The cDNA and predicted amino acid sequence of $L$. edodes cap is shown in Fig. 2 (the nucleotide sequence of the L. edodes cap gene has been submitted to DDBJ/ GeneBank/EMBL: accession number AB001578).

\section{Sequence comparison between $L$. edodes CAP and its homologues}

The L. edodes CAP shares high homology with other CAP homologues. It is $35.5 \%$ and $40.9 \%$ identical with Sacch. cerevisiae CAP and Schiz. pombe CAP, respectively, at the amino acid level. The identity between the C-terminal domain of L. edodes CAP and those of Sacch. cerevisiae and Schiz. pombe CAP is $39.0 \%$ and $46 \cdot 2 \%$, respectively, while the N-terminal domains of $L$. edodes CAP are $26.8 \%$ and $35.1 \%$ identical with the corresponding regions of Sacch. cerevisiae and Schiz. pombe CAP, respectively. So the C-terminal domain of 


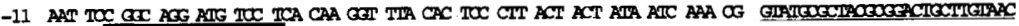
1 Met Ser Ser Gin Gly Leu His Ser Leu Thr Thr Ile Ile Iys

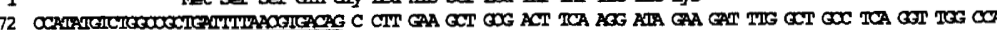
Anten Glu Ala

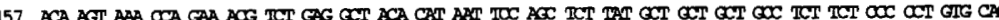
33 Thr Ser Iys Pro Gin Thr Ser Glu Ala Thr His Aen Ser Ser Ser tyr Ala Ala Ala Ala Ser Ser Pro Pro val Gln 33 Thr Ser Ijs Pro Glu Thr Ser Glu Ala Thr His An Ser Ser Ser Tyr Ala Ala Ala Ala Ser Ser P20 Pro Val Gin 59 Ala Ser Ser pro Ala Ala Pro Ala Ser Ala Ser Thr het Arg Gul Thr Pro Lys Ser val val val the Asp Giu Val

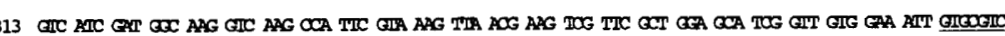
85 Val Ile Asp Gly IYs Val Irs Pro the Val Iys Ian Thr Ifys Ser the Ala Gly Ala Ser Val Val Glu Tle

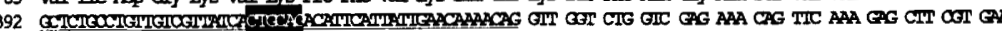

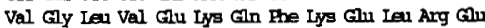

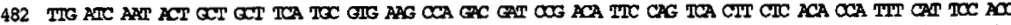
122 In Ile Aan thr Ala Ala Sar Cys Val Iys Pro Asp Aep Pro Thr the Gin Ser Lau Lau Thr Pro the his Ser Thr 560 TIC

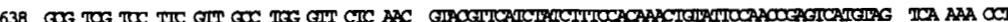
174 Ala sar Ser the Val Ala Tr Val Ial Aen Ser I prs Pro 174 Ala Ser Ser the Val Ala TrP Val Lau Aan

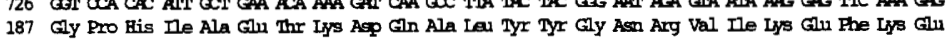

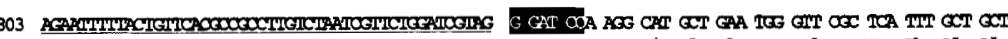
211 Iys Axp Pro Arg His Ala Glu Trp Val Ang Ser the Ala Ala 891 CCA TTA EAA GAG AOC COC AAG TAC GIC CTG GAG TAC CAT ACC ACT COA CIT TCA TGG AAC TOC AAA GIFCOICIGIOBCR 225 Ala Leu Gu Glu Thr Arg Iys Tyr Val Ieu Gu Tyr His Thr Thr Gy Leu Ser Trp Aen Ser Iys

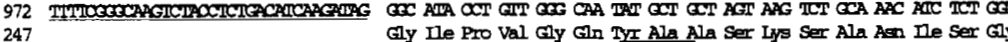
247

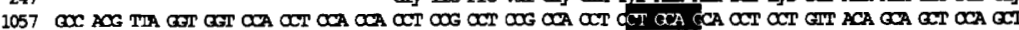
254 Ala thr Ian Gly Gly pro pro Pro pro pro Pro pro Pro Pro Pro Pro Ala Als Pro Pro Val thr Ala Ala Pro Ala

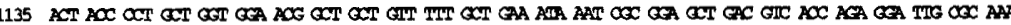
290 Thr Thr Pro Ala Gly Gly Ihr Ala Ala Val the Ala Gu Ile Aen Arg Gly Ala Asp Val thr Arg Gly Leu Arg Lys

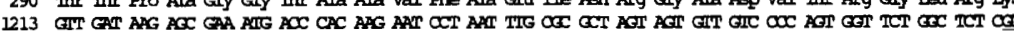
316 Val Asp lys Ser Glu Met Thr His Lse Aen Pro Aen Leu Arg Ala Ser Ser val val Pro Ser Gly Ser Gly Ser

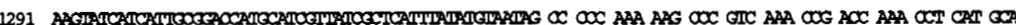
341

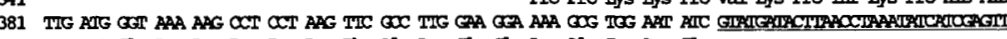
354 Leu Met Gly Lys Lys Pro Pro Lys the Ala Lai Glu Gly Lys Ala Trp Aen Ile

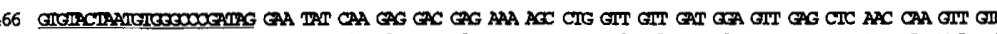
372 Gin Tyr Gin Glu Asp Glu Lys Ser Leu Val Val Asp Gly Val Glu Leu Aan Gln Val Val

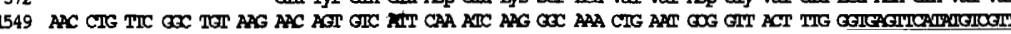

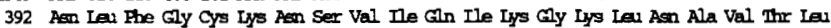

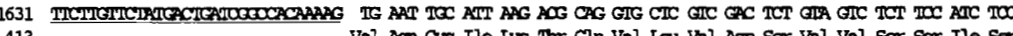

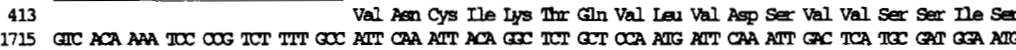
431 val Thr Iys Ser pro Ser the Ala Ile Gin De Thr Gy Ser Ain Pro bet lle Gln Ile Asp Ser Cya Asp Gly het

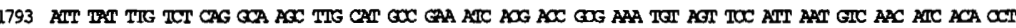
457 Ile Tyr Ial Ser Gin Ala Ser Ieu His Ala Gu Ile Thr Thr Ala Lys Cys Ser Ser Ile Aen Val Asn Tle Thr Pro

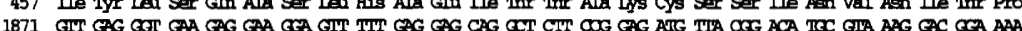
483 Val Glu Gly Glu Glu Glu Gly Val the Gu Gu Gln Ala Leu Pro Glu Mat Ieu Arg Thr cys Val Lys Aap Gly Iys

1949 CIG GIG ACC AOC GIA GIT GAG CAR GIA GET TAA TIA TIA TOC GIA CIA TRT RIA RIT CTG CAA GIG OCA TPC CIT TCA 509 Lai Val Thr Thr Val Val Glu His Val Gly *

2027 CAA CAT GA TRT TAT CES
Fig. 2. Sequences of $L$. edodes cap cDNA, genomic DNA and the encoded protein. The EcoRI sites at the two ends of the CDNA were derived from linkers used in cloning the cDNA. The CDNA encodes a protein of 518 aa residues. Numbers on the left indicate the nucleotides of the genomic DNA, numbered starting from the start codon, or the residue number of the first amino acid on that line. Thin underlines indicate introns, whose presence was deduced from a comparison of the genomic DNA sequence with the CDNA sequence. Thicker underlines indicate amino acid or nucleotide sequences used to design the primers for PCR. The internal two such sequences are the amino acid motifs from which degenerate oligonucleotide primers were designed to amplify a part of the cap gene. The primers corresponding to the nucleotide sequences at the $5^{\prime}$ and $3^{\prime}$ ends were designed to amplify the full-length cap. Two Pstl sites and one BamHI site in the nucleotide sequence are highlighted with a black background.
L. edodes CAP shows higher homology with yeast CAPs than does the $\mathrm{N}$-terminal domain. The alignment of the amino acid sequence of $L$. edodes CAP with the sequences of the two yeast CAPs is shown in Fig. 3. $L$. edodes CAP is approximately equidistantly related to human CAP and yeast CAPs. The phylogenetic tree of the amino acid sequences of $L$. edodes CAP and all known homologues of CAP is shown in Fig. 4.

\section{Amplification of the cap gene from the genomic DNA of $L$. edodes and identification of introns}

To establish the presence of introns, the cap gene on the genomic DNA was amplified by PCR using the primers Pfg and Prg. The genomic DNA was first digested with $K p n I$ or $X b a I$ and then used as a template for PCR. A PCR fragment of about $2 \cdot 1 \mathrm{~kb}$ was chosen, cloned into the pT7-Blue vector and sequenced. The sequence is shown in Fig. 2. Eight introns were found in the cap gene. There were no KpnI or extra BamHI sites located in the introns, but another PstI site was discovered in the second intron (nt 414-419).

\section{Southern hybridization}

To investigate whether L.edodes cap is a one-copy gene or a gene family, low-stringency Southern blotting was conducted using the full-length CDNA as a probe. Two bands appeared in the BamHI-digested sample $(2 \cdot 2$ and $8.0 \mathrm{~kb})$, one band appeared in the KpnI-digested sample $(20.0 \mathrm{~kb})$, and three bands $(0.6,2.0$ and $2.2 \mathrm{~kb})$ appeared in the PstI-digested sample (data not shown). This pattern of Southern hybridization fitted well with the results obtained by sequencing of the cap gene. Using low-stringency Southern hybridization, we were unable to detect another cap homologue from L.edodes. 


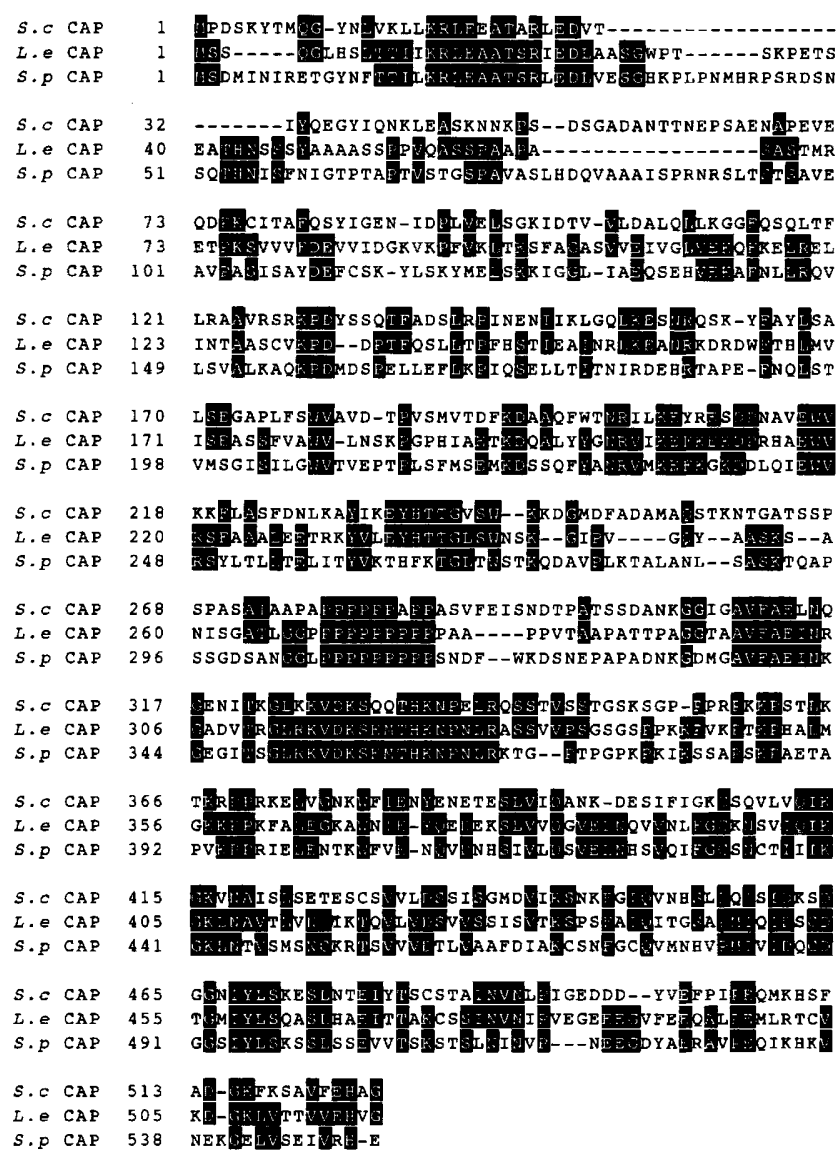

Fig. 3. Alignment of the amino acid sequences of $L$. edodes (L. e) CAP (accession number AB001578) with Sacch. cerevisiae (S. c) CAP (accession number P17555) and Schiz. pombe (S. p) CAP (accession number P36621). $L$. edodes amino acid residues identical to the corresponding residues of either of the yeast CAPs together with the residues of the yeast CAPs are highlighted with a black background. Amino acid residues are numbered on the left. The spaces were manually introduced to maximize the alignment.

Expression of the cap gene during the development of $L$. edodes from a mycelium into a fruiting body

Total cellular RNA was isolated from primordia, immature stage I fruiting bodies, immature stage II fruiting bodies and mature fruiting bodies, and was subjected to Northern blot analysis using cap cDNA as a probe. As shown in Fig. 5, a clear hybridization signal of $1.7 \mathrm{~kb}$ (cap band) was detected in all the RNA blots with comparable intensity. To ensure equal loading and transfer of RNA, the radioactivity was removed from the blots and rehybridized with the cDNA clone $(1.2 \mathrm{~kb})$ of $L$. edodes ras, which has been shown to be transcribed at a constant level during development of the mycelium into a fruiting-body (Hori et al., 1991). As shown in Fig. 5 , a $1.2 \mathrm{~kb}$ ras signal was detected in all the RNA blots, again with equal intensity. These results show that the cap gene is also transcribed at a constant level during development of the mycelium into a fruiting body.

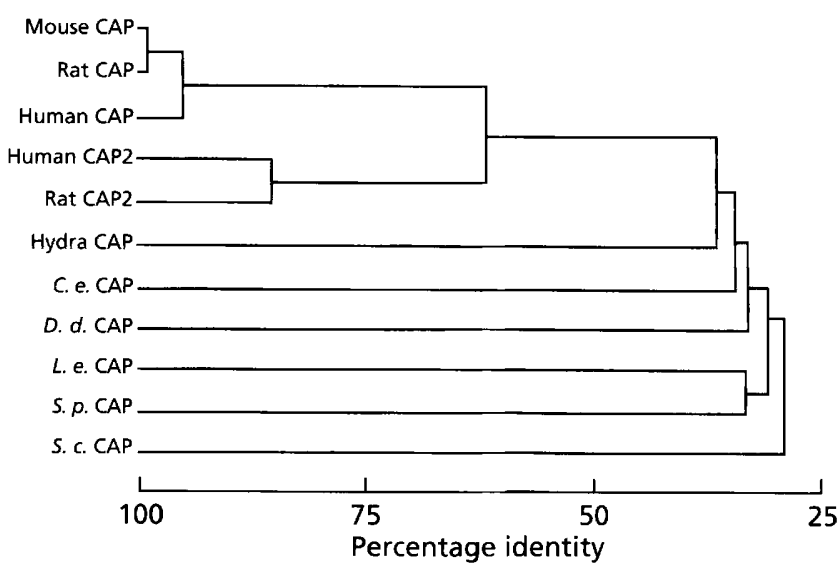

Fig. 4. A phylogenetic tree of the amino acid sequences of $L$. edodes CAP and other CAP homologues generated using the DNASIS software (Hitachi Software Engineering). The scale indicates the percentage identity as calculated from multiple alignments. The DNA or protein sequence accession numbers are: mouse CAP, P40124: rat CAP1, Q08163; human CAP1, Q01518; human CAP2, P40123; rat CAP2, P52481; hydra CAP, P40122; Caenorhabditis elegans (C. e.) CAP, AF000265; $D$. discoideum ( $D . \quad$ d.) CAP, P54654; $L$. edodes ( $L$. e.) CAP, AB001578; Schiz. pombe (S. p.) CAP, P36621; Sacch. cerevisiae (S. C.) CAP, P17555.

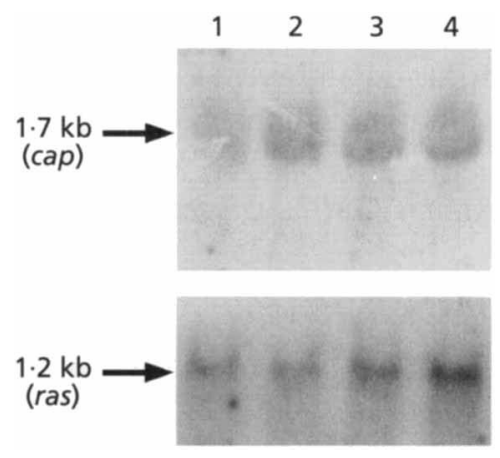

Fig. 5. Transcriptional expression of the cap gene during development of the mycelium into a fruiting body in $L$. edodes. Total cellular RNAs isolated from the primordia (lane 1), immature fruiting bodies [stage I: just after the formation of pileus and stipe], (lane 2), immature fruiting bodies [stage II: fruiting bodies are enlarged but gill tissues are not yet visible] (lane 3), and mature fruiting bodies (lane 4) were subjected to Northern-blot analysis using the ${ }^{32}$ p-labelled cap cDNA fragment as a probe (top). The label was removed from the blots by boiling in $0.1 \%$ SDS. After confirming the removal of radioactivity, the resulting blots were rehybridized with ${ }^{32} p_{-}$ labelled $L$. edodes ras CDNA (Hori et al., 1991) (bottom).

\section{Complementation of Cap- phenotypes in yeast by expression of the $L$. edodes CAP}

In Sacch. cerevisiae, loss of the C-terminal functional domain of CAP results in abnormal cellular morphology (round and enlarged cells), and growth impairment on rich medium (YPD), on synthetic medium (SC) containing $6.5 \mathrm{mM}$ valine and at $37^{\circ} \mathrm{C}$ (Gerst et al., 1991). 


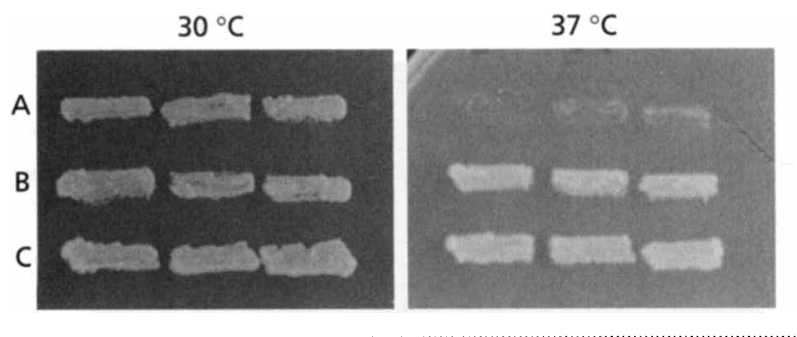

Fig. 6. Expression of $L$. edodes CAP suppresses the temperaturesensitive phenotype of the Schiz. pombe cap strain MK1818d. Cells were grown on PMA plates, replica-plated onto fresh PMA plates, and then incubated at $30^{\circ} \mathrm{C}$ or $37^{\circ} \mathrm{C}$. A, Schiz. pombe strain MK1818d transformed with pART1 (expression vector); B, MK1818d transformed with PPT1CAP, a plasmid expressing $L$. edodes CAP; C, MK1818d transformed with PCGE2, a plasmid expressing Schiz. pombe CAP. Three independent transformants are shown for each treatment.

In Schiz. pombe, deletion of the C-terminal functional domain also results in abnormal cellular morphology, growth impairment at $37^{\circ} \mathrm{C}$ and abnormalities in mating and sporulation. To see if it is possible to functionally complement a Schiz. pombe cap mutant with L. edodes CAP, we constructed the plasmid pPT1CAP and expressed it in the Schiz. pombe cap strain MK1818d (see Methods). Expression of the $L$. edodes CAP in Schiz. pombe restored its ability to grow at $37^{\circ} \mathrm{C}$ (Fig. 6), restored normal cellular morphology, and restored the capability of cells to sporulate when grown on minimum medium (PMA plates) (Fig. 7).

To investigate the possibility of functional conservation between L. edodes CAP and Sacch. cerevisiae CAP, we (a)

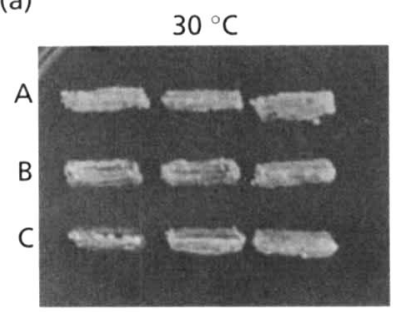

(b)

SC-Leu + Val
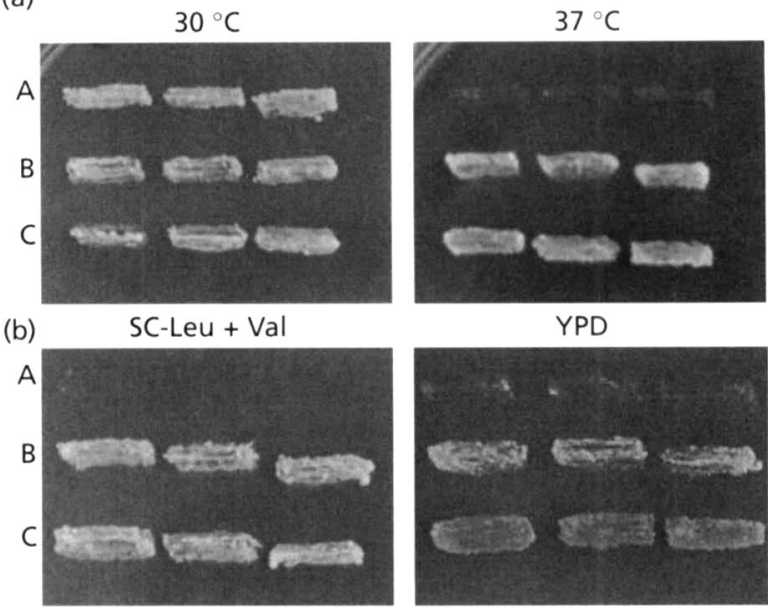

YPD

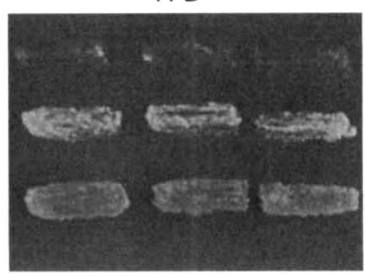

Fig. 8. Expression of $L$. edodes CAP suppresses the temperaturesensitive phenotype and inability to grow on rich medium of the Sacch. cerevisiae cap strain SKN32. Cells were grown on SC plates containing appropriate auxotrophic supplements and replica-plated onto various media. For each treatment, three independent transformants are shown. (a) SKN32 transformed with pAD5 (control vector; A), pPD5CAP (a plasmid expressing $L$. edodes CAP; B), or PADHCAP (a plasmid expressing Sacch. cerevisiae (AP; C), was replica-plated onto SC-Leu plates and incubated at $30^{\circ} \mathrm{C}$ or $37^{\circ} \mathrm{C}$. (b) SKN32 transformed with the same three plasmids as in (a) was replica-plated onto SC-Leu medium containing $6.5 \mathrm{mM}$ valine (left) or YPD rich medium (right) and incubated at $30^{\circ} \mathrm{C}$.

constructed the plasmid pPD5CAP and expressed the $L$. edodes CAP in the Sacch. cerevisiae cap strain SKN32 (see Methods). The expression of $L$. edodes CAP
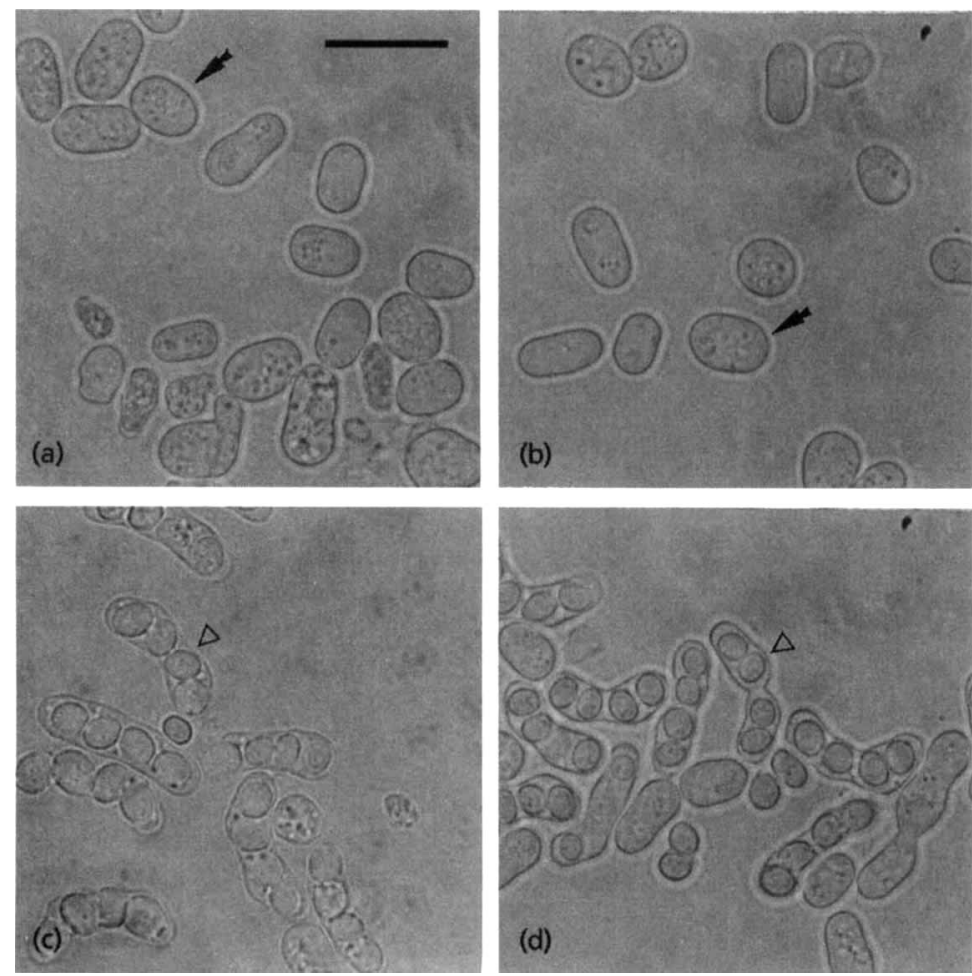

Fig. 7. Expression of $L$. edodes CAP restores the cellular morphology and sporulation of the Schiz. pombe cap strain MK1818d. Cells grown for $2 \mathrm{~d}$ on plates of minimal medium PMA were dispersed in distilled water and photographed. (a) MK1818d, in which the cap gene has been replaced with the ura4 gene; (b) MK1818d transformed with the control plasmid, Schiz. pombe expression vector PART1; (c) MK1818d transformed with PPT1CAP, a plasmid expressing $L$. edodes CAP; (d) MK1818d transformed with pCGE2, a plasmid expressing Schiz. pombe CAP. In (a) and (b), enlarged and round cells (black arrows) are abundant, and the cells do not sporulate. In (c) and (d), normal-sized and sporulating cells (open arrowheads) are abundant. Bar $10 \mu \mathrm{m}$. 

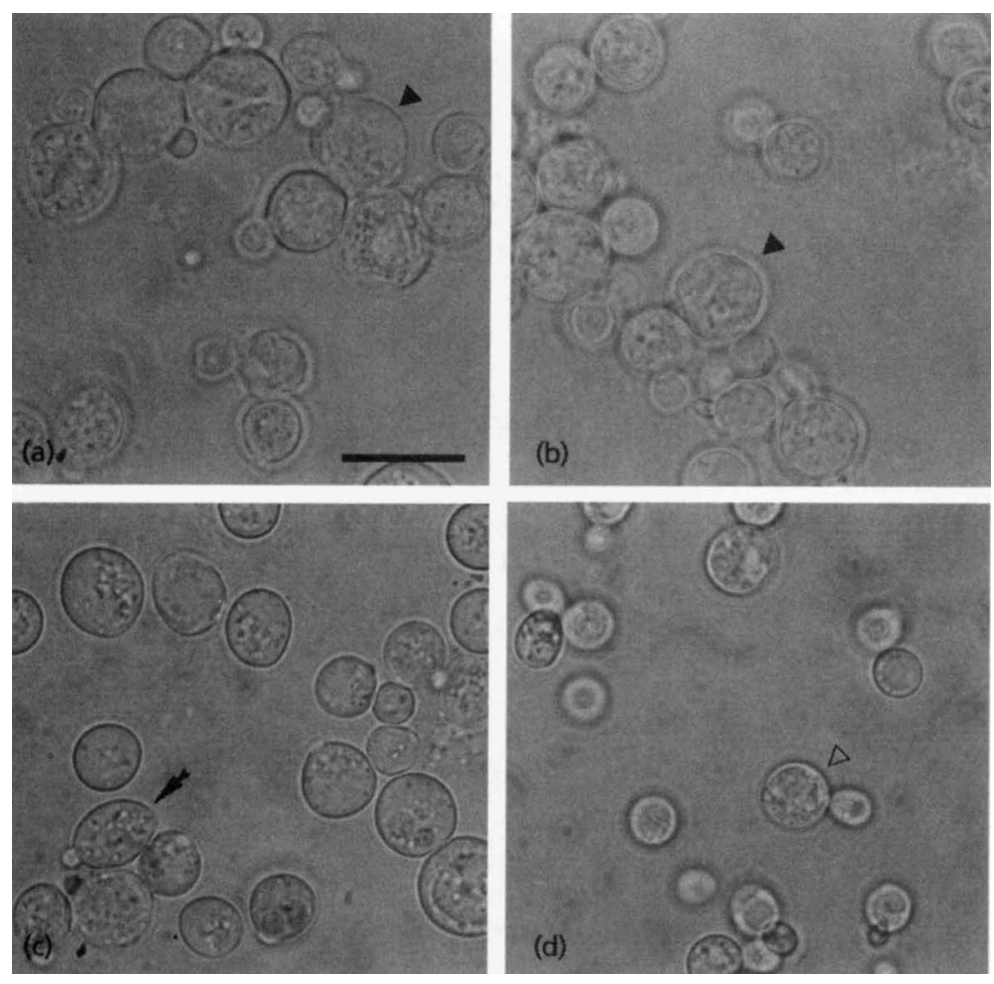

Fig. 9. Cellular morphology of the Sacch. cerevisiae cap strain SKN32 and transformants expressing various plasmids. Cells grown for $2 \mathrm{~d}$ on on SC plates without the selective supplements were dispersed in water and photographed. (a) SKN32, in which the cap gene has been replaced with the HIS3 gene; (b) SKN32 transformed with the control plasmid, Sacch. cerevisiae expression vector PAD5; (c) SKN32 transformed with PPD5CAP, a plasmid expressing $L$. edodes CAP; (d) SKN32 transformed with PADHCAP, a plasmid expressing Sacch. cerevisiae CAP. In (a) and (b), enlarged and round cells with a disordered surface (black arrowheads) are abundant. In (d), the cell shape and size are restored (open arrowhead). In the cells expressing $L$. edodes CAP (c), the cell shape is restored to some extent (cells are not so round and do not appear to have a disordered surface as in a and b) (black arrow). Bar $10 \mu \mathrm{m}$. restored the ability to grow on rich medium (YPD) and on SC medium containing a high concentration of valine, and produced normal growth at $37^{\circ} \mathrm{C}$. The degree of restoration of the normal phenotype was similar to that seen in the mutant cells transformed with the plasmid expressing Sacch. cerevisiae CAP (Fig. 8). The cell shape was restored to some extent even though there were still some abnormal cells (Fig. 9). This might be due to the low expression level of $L$. edodes CAP in SKN32 cells.

\section{Yeast two-hybrid assay}

Previous results from biochemical and yeast two-hybrid assays have indicated that yeast and human CAP interact with actin in vivo (Amberg et al., 1995; Freeman et al., 1995; Hubberstey et al., 1996). The L. edodes CAP can complement the morphological function of Schiz. pombe CAP. We therefore speculated that the L. edodes CAP should be able to interact with Schiz. pombe actin in vivo, thus enabling the normal formation of Schiz. pombe actin filaments. We performed the yeast twohybrid system assay to test this hypothesis. We first constructed pBTMCAP, an expression plasmid that expresses a LexA-fused $L$. edodes CAP protein (see Methods), and used this plasmid to co-transform Sacch. cerevisiae strain L 40 with plasmid pGADAC, which has the Schiz. pombe actin gene cloned into pGADGH, where it is orientated in the correct frame to allow expression. As negative controls, the cells were simultaneously co-transformed with combinations of pGADAC and pBTMmsa2 along with pBTMCAP and pGADGb. The $m s a 2$ gene encodes an RNA-binding protein and the gbd1 gene encodes $\mathrm{G} \beta$; these two proteins are unrelated to either CAP or actin. As a positive control, CAP and actin of Schiz. pombe, two proteins known to interact with each other in vivo, were similarly co-expressed in Sacch. cerevisiae strain L40 and $\beta$-galactosidase activity was measured. The $L$. edodes CAP was found to interact with Schiz. pombe actin as indicated by the appearance of blue-coloured colonies. However, judging by the intensity of the blue colour, the interaction seemed to be weaker than that of the positive control (results not shown).

\section{DISCUSSION}

CAP is an interesting protein that is involved in signal transduction and cytoskeleton formation, which are two important processes in a wide range of organisms. We have identified a CAP homologue from the mushroom L. edodes and analysed its characteristics. Comparison of the amino acid sequence of $L$. edodes CAP with those of other CAP homologues indicated that it is nearly equidistantly related to homologues from other organisms, and that the homology with other CAPs is about $30 \%$. The homology among mammalian CAPs is extremely high (Fig. 4), in contrast to CAP homologues from Sacch. cerevisiae and Schiz. pombe, which share little homology. This is consistent with the notion that these two yeast species are not as closely related as their common name - yeast - would appear to suggest. The L. edodes CAP homologue displays the highest degree of homology with Schiz. pombe CAP, with an identity of $40.9 \%$, while it shows $35.5 \%$ identity with Sacch. 
cerevisiae CAP. The C-terminal domain and the central domain also tended to be well-conserved between $L$. edodes CAP and the two yeast CAPs compared to the degree of conservation between the $\mathrm{N}$-terminal domains of yeast and mushroom CAPs. The RLE repeat localized in Sacch. cerevisiae CAP at amino acids 19-21 and 26-28, which is conserved in most homologues, can potentially form an amphipathic helix suitable for protein-protein interaction (Vojtek \& Cooper, 1993). This repeat was altered to RLE/RIE (aa 15-17 and 22-24) in L. edodes CAP. The proline-rich stretch, which is presumed to be the $\mathrm{SH} 3$ protein binding motif (Freeman et al., 1995), contained 11 consecutive proline residues from amino acids 269 to 279 in mushroom CAP. This is the longest stretch of proline among all the CAP homologues identified so far. We were unable to detect another CAP homologue in L. edodes by lowstrigency Southern blotting. In addition, the cap gene is likely to be transcribed at similar levels during the development of $L$. edodes from a mycelium into a fruiting-body. From the genomic sequences, eight introns were identified. This is the first time that introns have been identified in a CAP-homologous gene. The fact that CAP proteins have been conserved during the evolution from single-cell eukaryote yeasts to humans implies that CAP fulfils essential cellular functions. The CAP of L. edodes is more homologous to Schiz. pombe CAP than to Sacch. cerevisiae CAP. Expression of the $L$. edodes CAP in the Schiz. pombe cap strain MK1818d fully complemented the defects resulting from the loss of the C-terminal domain of the endogenous CAP. The expression of $L$. edodes CAP in the Sacch. cerevisiae cap strain SKN32 suppressed some of the defects related to the deletion of endogenous CAP, but the restoration of cell size and morphology was incomplete. We speculate that this may be due to the insufficient level of expression of L. edodes CAP.

An interaction between CAP and actin has been suggested by previous genetic studies on yeast CAP (Vojtek et al., 1991; Amberg et al., 1995) and by the copurification of porcine CAP with actin (Gieselman \& Mann 1992). Yeast CAP was suggested to be required for normal actin filament formation. Recent studies show that yeast CAP interacts with monomeric actin and thus prevents it from polymerizing in vitro (Freeman et al., 1995). This interaction requires the C-terminal domain, but not the $\mathrm{N}$-terminal domain, of CAP (Hubberstey et al., 1996). Using the yeast two-hybrid system, the conservation of the interaction between CAP and actin in yeast and mammal was verified (Amberg et al., 1995; Freeman et al., 1995; Hubberstey et al., 1996). Our functional complementation analysis result showed that $L$. edodes CAP can fully complement the morphological defects of Schiz. pombe cap strains, and the results of the two-hybrid assay indicate that $L$. edodes CAP interacts with Schiz. pombe actin in vivo. These results provide convincing evidence that CAP homologues from other organisms can suppress the morphological defects associated with the cap mutation in yeast cells.
Although many CAP homologues have been cloned, only yeast CAPs have been functionally identified as adenylyl-cyclase-associated proteins; the identification of others has only been based on their homology and conservation of the C-terminal domain functions with yeast CAPs. It will be interesting to see if $L$. edodes CAP does indeed function as an adenylyl-cyclase-associated protein.

\section{ACKNOWLEDGEMENTS}

This work was supported by a Grant-in-Aid from the Kato Bioscience Foundation and the Ministry of Education, Science and Culture of Japan. T. Yamamoto participated in some of the work.

\section{REFERENCES}

Amberg, D. C., Basart, E. \& Botstein, D. (1995). Defining protein interactions with yeast actin in vivo. Nat Struct Biol 2, 28-35.

Ballester, R., Michaeli, T., Ferguson, K., Xu, P., McCormick, F. \& Wigler, M. (1989). Genetic analysis of mammalian GAP expressed in yeast. Cell 59, 681-686.

Bartel, P. L., Chien, C., Sternglanz, R. \& Fields, S. (1993). Using the two-hybrid system to detect protein-protein interactions. In Cellular Interactions in Development: a Practical Approach, pp. 153-179. Edited by D. A. Hartley. Oxford: IRL Press.

Fedor-Chaiken, M., Deschenes, R. J. \& Broach, J. R. (1990). SR V2, a gene required for $R A S$ activation of adenylate cyclase in yeast. Cell 61, 329-340.

Fenger, U., Hofmann, M., Galliot, B. \& Schaller, H. C. (1994). The role of the cAMP pathway in mediating the effect of head activator on nerve-cell determination and differentiation in hydra. Mech Dev 47, 115-125.

Field, J., Vojtek, A., Ballester, R., Bolger, G., Colicelli, J., Ferguson, K., Gerst, J., Kataoka, T., Michaeli, T., Powers, S., Riggs, M. Rodgers, L., Wieland, I., Wheland, B. \& Wigler, M. (1990). Cloning and characterization of CAP, the $S$. cerevisiae gene encoding the $70 \mathrm{kd}$ adenylyl cyclase-associated protein. Cell 61, 319-327.

Fields, S. \& Song, O. (1989). A novel genetic system to detect protein-protein interactions. Nature 340, 245-246.

Freeman, N. L., Chen, Z., Horenstein, J., Weber, A. \& Field, J. (1995). An actin monomer binding activity localizes to the carboxyl-terminal half of the Saccharomyces cerevisiae cyclaseassociated protein. J Biol Chem 270, 5680-5685.

Freeman, N. L., Lila, T., Mintzer, K. A., Chen, Z., Pahk, A. J., Ren, R., Drubin, D. G. \& Field, J. (1996). A conserved proline-rich region of the Saccharomyces cerevisiae cyclase-associated protein binds SH3 domains and modulates cytoskeletal localization. Mol Cell Biol 16, 548-556.

Gerst, J. E., Ferguson, K., Vojtek, A., Wilger, M. \& Field, J. (1991). CAP is a bifunctional component of the Saccharomyces cerevisiae adenylyl cyclase complex. Mol Cell Biol 11, 1248-1257.

Gieselmann, R. \& Mann, K. (1992). ASP-56, a new actin sequestering protein from pig platelets with homology to CAP, an adenylate cyclase-associated protein from yeast. FEBS Lett 298 , 149-153.

Gottwald, U., Brokamp, R., Karakesisoglou, I., Schleicher, M. \& Noegel, A. A. (1996). Identification of a cyclase-associated protein 
(CAP) homologue in Dictyostelium discoideum and characterization of its interaction with actin. Mol Biol Cell 7, 261-272.

Han, J. H., Stratowa, C. \& Rutter, W. J. (1987). Isolation of fulllength putative rat lysophospholipase cDNA using improved methods for mRNA isolation and cDNA cloning. Biochemistry 26, 1617-1625.

Hori, K., Kajiwara, S., Saito, T., Miyazawa, H., Katayose, Y. \& Shishido, K. (1991). Cloning, sequence analysis and transcriptional expression of a ras gene of edible basidiomycete Lentinus edodes. Gene 105, 91-96.

Hubberstey, A., Yu, G., Loewith, R., Lakusta, C. \& Young, D. (1996). Mammalian CAP interacts with CAP, CAP2, and actin. J Cell Biochem 60, 459-466.

Huynh, T. V., Young, R. A. \& Davis, R. W. (1985). Construction and screening cDNA libraries in $\lambda \mathrm{gt} 10$ and $\lambda \mathrm{gt} 11$. In DNA Cloning: a Practical Approach, vol. 1, pp. 49-72. Edited by D. M. Glover. Oxford: IRL Press.

Ito, H., Fukuda, Y., Murata, K. \& Kimura, A. (1983). Transformation of intact yeast cells treated with alkali cations. $J$ Bacteriol 153, 163-168.

Kawamukai, M., Gerst, J., Field, J., Riggs, M., Rodgers, L., Wigler, M. \& Young, D. (1992). Genetic and biochemical analysis of the adenylyl cyclase-associated protein, cap, in Schizosaccharomyces pombe. Mol Biol Cell 3, 167-180.

Mcleod, M., Stein, M. \& Beach, D. (1987). The product of the $m e i 3^{+}$gene, expressed under control of the mating-type locus, induces meiosis and sporulation in fission yeast. $E M B O J \mathbf{5}$, $3665-3671$.

Marcus, S., Wigler, M., Xu, P., Ballester, R., Kawamukai, M. \& Polverim, A. (1993). RAS function and protein kinase cascades. Ciba Found Symp 176, 53-61.

Matviw, H., Yu, G. \& Young, D. (1992). Identification of a human cDNA encoding a protein that is structurally and functionally related to the yeast adenylyl cyclase-associated CAP proteins. Mol Cell Biol 12, 5033-5040.

Mintzer, K. A. \& Field, J. (1994). Interaction between adenyly cyclase, CAP and RAS from Saccharomyces cerevisiae. Cell Signalling 6, 681-694.

Mitchison, J. M. (1970). Physiological and cytological methods for Schizosaccharomyces pombe. Methods Cell Physiol 4, 131-165.

Rose, M. D., Winston, F. \& Hieter, P. (1990). Methods in Yeast Genetics: a Laboratory Course Manual. Cold Spring Harbor, NY: Cold Spring Harbor Laboratory.
Sambrook, J., Fritsch, E. F. \& Maniatis, T. (1989). Molecular Cloning: a Laboratory Manual, 2nd edn. Cold Spring Harbor, NY: Cold Spring Harbor Laboratory.

Sanger, F., Nicklen, S. \& Coulson, A. R. (1977). DNA sequencing with chain-terminating inhibitors. Proc Natl Acad Sci USA 74, 5463-5467.

Shima, F., Yamawaki-Kataoka, Y., Yanagihara, C., Tamada, M., Okada, T., Kariya, K. \& Kataoka, T. (1997). Effect of association with adenylyl cyclase-associated protein on the interaction of yeast adenylyl cyclase with Ras protein. Mol Cell Biol 17, 1057-1064.

Swiston, J., Hubberstey, A., Yu, G. \& Young, D. (1995). Differential expression of CAP and CAP2 in adult rat tissues. Gene 165, 273-277.

Takagi, Y., Katayose, Y. \& Shishido, K. (1988). Intracellular levels of cyclic AMP and adenylate cyclase activity during mycelial development in fruiting body formation in Lentinus edodes. FEMS Microbiol Lett 55, 257-278.

Vojtek, A. B. \& Cooper, J. A. (1993). Identification and characterization of a cDNA encoding mouse CAP: a homolog of the yeast adenylyl cyclase associated protein. J Cell Sci 105, 777-785.

Vojtek, A., Haarer, B., Field, J., Gerst, J., Pollard, T. D., Brown, S. \& Wigler, M. (1991). Evidence for a functional link between profilin and CAP in the yeast $S$. cerevisiae. Cell 66, 497-505.

Wang, J., Suzuki, N. \& Kataoka, T. (1992). The 70-kilodalton adenylyl cyclase-associated protein is not essential for interaction of Saccharomyces cerevisiae adenylyl cyclase with RAS proteins. Mol Cell Biol 12, 4937-4945.

Yu, G., Swiston, J. \& Young, D. (1994). Comparison of human CAP and CAP2, homologs of the yeast adenylyl cyclase-associated proteins. J Cell Sci 107, 1671-1678.

Zelicof, A., Gatica, J. \& Gerst, J. E. (1993). Molecular cloning and characterization of a rat homolog of CAP, the adenylyl cyclaseassociated protein from Saccharomyces cerevisiae. J Biol Chem 268, 13448-13453.

Zelicof, A., Protopopov, V., David, D., Lin, X. Y., Lustgarten, V. \& Gerst, J. E. (1996). Two separate functions are encoded by the carboxyl-terminal domains of the yeast cyclase-associated protein and its mammalian homologs. J Biol Chem 271, 18243-18252.

Zolan, M. E. \& Pukkila, P. J. (1986). Inheritance of DNA methylation in Coprinus cinereus. Mol Cell Biol 6, 195-200.

Received 3 October 1997; revised 4 December 1997; accepted 24 December 1997. 